\title{
Physics of collisionless reconnection in a stressed X-point collapse
}

\author{
D. Tsiklauri and T. Haruki \\ Joule Physics Laboratory, Institute for Materials Research, \\ University of Salford, Manchester, M5 4WT, United Kingdom
}

(Dated: November 7, 2018)

\begin{abstract}
Recently, magnetic reconnection during collisionless, stressed, X-point collapse was studied using kinetic, 2.5D, fully electromagnetic, relativistic Particle-in-Cell numerical code [D. Tsiklauri and T. Haruki, Phys. Plasmas 14, 112905 (2007)]. Here we finalise the investigation of this topic by addressing key outstanding physical questions: (i) which term in the generalised Ohm's law is responsible for the generation of the reconnection electric field? (ii) how does the time evolution of the reconnected flux vary with the ion-electron mass ratio? (iii) what is the exact energy budget of the reconnection process, i.e. in which proportion initial (mostly magnetic) energy is converted into other forms of energy? (iv) are there any anisotropies in the velocity distribution of the accelerated particles? It has been established here that: (i) reconnection electric field is generated by the electron pressure tensor off-diagonal terms, resembling to the case of tearing unstable Harris current sheet studied by the GEM reconnection challenge; (ii) For $m_{i} / m_{e} \gg 1$ the time evolution of the reconnected flux is independent of ion-electron mass ratio; also, in the case of $m_{i} / m_{e}=1$ we show that reconnection proceeds slowly as the Hall term is zero; when $m_{i} / m_{e} \gg 1$ (i.e. the Hall term is non-zero) reconnection is fast and we conjecture that this is due to magnetic field being frozen into electron fluid, which moves significantly faster than ion fluid; (iii) within one Alfvén time, somewhat less than half $(\sim 40 \%)$ of the initial total (roughly magnetic) energy is converted into the kinetic energy of electrons, and somewhat more than half $(\sim 60 \%)$ into kinetic energy of ions (similar to solar flare observations); (iv) in the strongly stressed X-point case, in about one Alfvén time, a full isotropy in all three spatial directions of the velocity distribution is seen for super-thermal electrons (also commensurate to solar flare observations).
\end{abstract}

PACS numbers: 52.35.Vd; 96.60.Iv; 52.65.Rr; 45.50.Dd; 96.60.pf; 96.60.qe

\section{INTRODUCTION}

In many astrophysical or laboratory plasma situations (a) plasma beta is small, indicative of large amounts of energy stored in a form of magnetic field and (b) there is a need to explain or provide plasma heating, as well as plasma particle acceleration. It is believed that in such situations magnetic reconnection, i.e. change of connectivity of magnetic field lines that penetrate the plasma, can serve as one of the important possible mechanisms. There are different types of magnetic reconnection. One of the key descriptors is plasma collisionality, i.e. if plasma is collisional then magnetic resistivity, $\eta$ (or more specifically $\eta \vec{j}$ term in the generalised Ohm's law) is responsible for breaking the frozen-in condition (enabling field line connectivity change). However, if plasma is collisionless, then other terms in the generalised Ohm's law may be more important. In this context, it is instructive to look at typical spatial scales. Let us consider an example of solar coronal plasma. Typical width of a Sweet-Parker current sheet is given by $\delta=S^{-1 / 2} L[1]$ (p. 54). Fixing coronal temperature at $1 \mathrm{MK}$, Coulomb logarithm at 18.0948, the Lundquist number (using Spitzer resistivity from Huba [2], p. 30) is $5.37933 \times 10^{12}$, which for $L=10 \mathrm{Mm}$ yields, $\delta=4.31157 \mathrm{~m}$. Another way of looking at $\delta$ is associating it also with the resistive length scale via Alfvén time scale $\tau_{A}=L / V_{A}$, where $V_{A}$ is the Alfvén speed $\left(\approx 1 \mathrm{Mm} \mathrm{s}^{-1}\right)$ : i.e. $\delta=S^{-1 / 2} L=\eta \tau_{A} / \mu_{0}$. Typical scale associated with the Hall term in the generalised Ohm's law at which deviation from electron-ion coupled dynamics is observed is, $c / \omega_{p i}=7.20064 \mathrm{~m}$ (ion inertial length). Here particle density of $n=10^{15} \mathrm{~m}^{-3}$ is used. The fact that $\delta /\left(c / \omega_{p i}\right) \leq 1$ points to a necessity of going beyond single fluid MHD approximation. The Hall term itself cannot break the frozen-in condition, its inclusion into consideration ensures that the magnetic field is frozen into electron fluid. Below, we shall use this argument to conjecture why reconnection is fast when the Hall term is included. Importance of different terms in the generalised Ohm's law is usually inferred by comparing the spatial scales associated with them to the resistive length scale $\delta$. E.g. one of the other noteworthy scales is $c / \omega_{p e}=0.16804 \mathrm{~m}$ (electron inertial length) on which the electron inertia term operates. When $\delta /\left(c / \omega_{p e}\right)<1$ then electron inertia would dominate over resistive diffusion [1] (p. 200). As can be seen from the above estimates $\delta /\left(c / \omega_{p e}\right)=25.6576 \gg 1$ in the solar corona, thus electron inertia effects seem to be negligible. However, electron inertia in contrary to the Hall term (as well as the electron pressure tensor) can break the frozen-in condition and thus change the magnetic field connectivity. Because, of the fact that with increase of $T$ (hot plasmas), $\delta$ gets progressively smaller thus effects other than the resistivity should be included. Indeed, collisionless (non-resistive) reconnection has recently attracted considerable attention (see Birn and Priest [3] for a review).

One of the first studies of magnetic reconnection is stressed, X-point collapse [4] (also see Chap. 7.1 in Priest and Forbes [5]). The latter was using resistive MHD approach. We recently revisited the problem in the regime 
of collisionless reconnection [ [6]. In Ref. [ [ ] $]$ we studied the magnetic reconnection during collisionless, stressed, Xpoint collapse using kinetic, $2.5 \mathrm{D}$, fully electromagnetic, relativistic Particle-in-Cell numerical code. We considered two cases of weakly and strongly stressed X-point collapse. Where descriptors weakly and strongly refer to $20 \%$ and $124 \%$ unidirectional spatial compression of the X-point, respectively. Amongst other interesting outcomes, we established that within about one Alfvén time, $2 \%$ and $20 \%$ of the initial magnetic energy can be converted into heat and accelerated particle energy in the cases of weak and strong stress, respectively. However, open questions remained: (i) which term in the generalised Ohm's law is responsible for the generation of the reconnection electric field? (ii) how does the time evolution of the reconnected flux vary with the ion-electron mass ratio? (iii) what is the exact energy budget of the reconnection process, i.e. in which proportion initial (mostly magnetic) energy is converted into other forms of energy? (iv) are there any anisotropies in the velocity distribution of the accelerated particles? Here we finalise the study of magnetic, collisionless reconnection of a stressed X-point by providing answers to these questions.

\section{THE MODEL}

The numerical code used here is $2.5 \mathrm{D}$, relativistic, fully electromagnetic PIC code, with the initial conditions the same as in our previous work [6]. For completeness we re-iterate key points: Magnetic field configuration is an $\mathrm{X}$-point without a guide-field

$$
\left(B_{x}, B_{y}, B_{z}\right)=\frac{B_{0}}{L}\left(y, \alpha^{2} x, 0\right),
$$

where $B_{0}$ is magnetic field intensity at the distance $L$ from the $\mathrm{X}$-point ( $L$ is the global system scale). $\alpha$ is the stress parameter, which prescribes the initial strength of magnetic pressure that collapses the system, due to lack of restoring force [5]. Using $\mu_{0} \vec{j}=\nabla \times \vec{B}$, a uniform current is imposed in the $z$ direction,

$$
j_{z}=\frac{B_{0}}{\mu_{0} L}\left(\alpha^{2}-1\right) .
$$

Electrons and ions have uniform spatial, and Maxwellian velocity distributions throughout the system. For $\alpha=1$, magnetic field geometry is completely symmetric, $j_{z}$ current zero (see Eqs. (11)-(2)), and thus, such magnetic configuration is stable. For $\alpha>1$, stressed X-point starts collapse in the $x$ direction because of the absence of a restoring force, causing time-transient magnetic reconnection. The main parameters of the standard simulation model are as follows. The length of the system in two dimensions is $L_{x}=L_{y}=400 \Delta$ (this is excluding so-called ghost cells), where $\Delta=1$ is the simulation grid size corresponding to electron Debye length,
$\lambda_{D}=v_{t e} / \omega_{p e}=1 \Delta\left(v_{t e}\right.$ is electron thermal velocity and $\omega_{p e}$ is electron plasma frequency). The global reconnection scale is set $L=200 \Delta$. The number density is fixed at $n_{0}=100$ electron-ion pairs per cell. Hence the total number is $1.6 \times 10^{7}$ pairs. The simulation time step is $\omega_{p e} \Delta t=0.05$. Ion-to-electron mass ratio is fixed at $m_{i} / m_{e}=100$ (which is varied in Fig. 2 (subsection III.B) only). The electron thermal velocity to speed of light ratio is $v_{t e} / c=0.1$. The electron and ion skin depths are $c / \omega_{p e}=10 \Delta$ and $c / \omega_{p i}=100 \Delta$, respectively. The electron cyclotron frequency to plasma frequency ratio is $\omega_{c e} / \omega_{p e}=1.0$ for magnetic field intensity, $B=B_{0}$. This ratio is close to unity in the solar corona, while it is much bigger than unity in the Earth magnetosphere. The electron and ion Larmor radii are $v_{t e} / \omega_{c e}=1 \Delta$ and $v_{t i} / \omega_{c i}=10 \Delta$, where $v_{t i}$ is the ion thermal velocity. Initial temperatures of electrons and ions are initially set the same, $T_{e}=T_{i}$. At the boundary $\left(B=B_{0}\right.$ at the distance $L$ from the X-point), the plasma $\beta=0.02$ and Alfvén velocity, $V_{A 0} / c=0.1$. Naturally these vary across the simulation box as the background magnetic field is a function of $x$ and $y$.

The boundary conditions on EM-fields are zerogradient and also, tangential component of electric field was forced to zero, while normal component of magnetic field was kept constant, both at the boundary. This ensures that there is no change in magnetic flux through the simulation box, i.e. the system is isolated. When colliding with boundaries particles are reflected. Thus our boundary conditions ensure there is no magnetic influx or mass transport across the boundaries. It has been also confirmed that the total energy in the system is conserved during the simulations to a good accuracy.

\section{RESULTS}

Before we address the outstanding questions, we refer reader to Tsiklauri and Haruki [6] for a more detailed description of dynamics of EM-fields, currents, and particles. It is our intention to focus on the outstanding questions here. In brief, the previous results can be summarised as follows: when $\alpha>1$, the stressed X-point collapses in the $x$ direction due to the absence of a restoring force, and hence time-transient magnetic reconnection occurs. The fast reconnection regime is achieved. Initially uniform out-of-plane current becomes localised, peaking at many times its initial value in a time transient manner. Also, out-of-plane quadruple magnetic field is generated. High energy part of the electron distribution function exhibits a power-law behaviour. Sizable fraction of initial magnetic energy is converted into other forms of energy. 

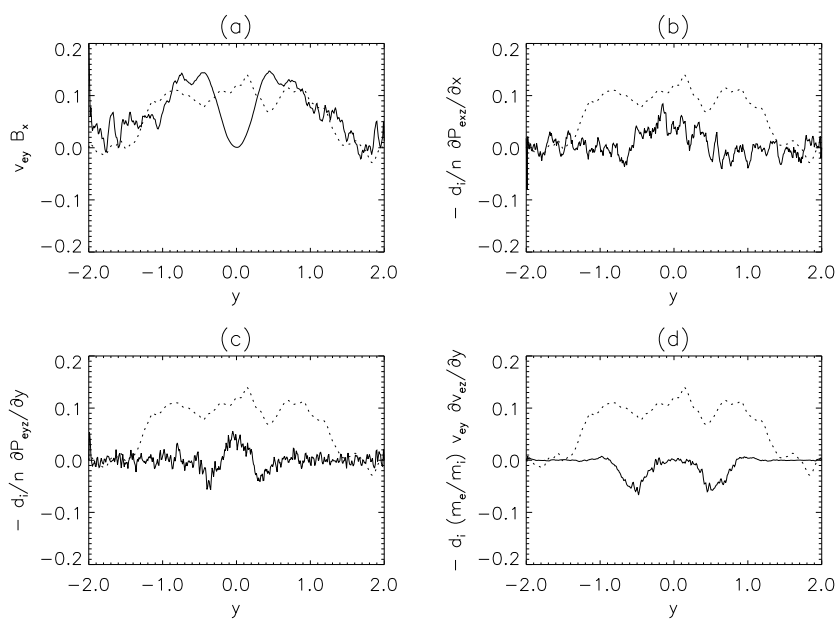

FIG. 1: Line plots of different terms in the generalised Ohm's law along $y$ direction, in $x=0$, at $\omega_{p e} t=170$ (time-transient reconnection peak) for $\alpha=1.20$. Solid lines in panels (a)-(d) show the different terms as follows: (a) $v_{e y} B_{x}$, (b) $-\left(d_{i} / n\right) \partial P_{e x z} / \partial x$, (c) $-\left(d_{i} / n\right) \partial P_{e y z} / \partial y$ and (d) $-d_{i}\left(m_{e} / m_{i}\right) v_{e y} \partial v_{e z} / \partial y$. The reconnection electric field $E_{z}(0, y)$ (normalised to $\left.V_{A 0} B_{0}\right)$ is shown with the dashed line in all four panels. Here $y$ is normalised to $c / \omega_{p i}$, and thus varies between $-2<y<2$.

\section{A. Source of the reconnection electric field}

In order to understand details of the collisionless reconnection process, we now focus on the question: which term in the generalized Ohm's law is responsible for the generation of the reconnection electric field? We adopt an appoach used by Pritchett [7]. The generalized Ohm's law can be written as (e.g. [3] p. 108)

$$
\vec{E}=-\vec{v}_{e} \times \vec{B}-\frac{\nabla \cdot \vec{P}_{e}}{n_{e} e}-\frac{m_{e}}{e}\left(\frac{\partial \vec{v}_{e}}{\partial t}+\left(\vec{v}_{e} \cdot \nabla\right) \vec{v}_{e}\right),
$$

where $\vec{E}$ and $\vec{B}$ are electric and magnetic fields, $\vec{v}$ is plasma velocity, $\vec{P}$ is pressure tensor $(3 \times 3$ matrix $), n$ is plasma number density, $m$ is mass and $e$ is electric charge. The subscript $e$ refers to an electron. Normalising space coordinates by global reconnection scale $L$, fluid velocity by Alfvén speed $V_{A}$, time by Alfvén transit time $\tau_{A}\left(=L / V_{A}\right)$, magnetic field by $B_{0}$, number density by $n_{0}$ and pressure tensor by $B_{0}^{2} / \mu_{0}$, a dimensionless version of Eq.(3) can be obtained

$$
\vec{E}=-\vec{v}_{e} \times \vec{B}-d_{i} \frac{\nabla \cdot \vec{P}_{e}}{n_{e}}-d_{i} \frac{m_{e}}{m_{i}}\left(\frac{\partial \vec{v}_{e}}{\partial t}+\left(\vec{v}_{e} \cdot \nabla\right) \vec{v}_{e}\right),
$$

where $d_{i}$ is the normalised ion skin depth $\left(d_{i}=c / \omega_{p i} L\right)$. Note that strictly speaking we should have used tildes in Eq.(44) to denote dimensionless quantities, but we omit them for brevity.

Let us focus on the out-of-plane component of the electric field $E_{z}$ at the magnetic null, which is a measure of the reconnection rate. It is given by,

$$
\begin{aligned}
E_{z}= & -\left(v_{e x} B_{y}-v_{e y} B_{x}\right)-d_{i} \frac{1}{n}\left(\frac{\partial P_{e x z}}{\partial x}+\frac{\partial P_{e y z}}{\partial y}\right) \\
& -d_{i} \frac{m_{e}}{m_{i}}\left(\frac{\partial v_{e z}}{\partial t}+v_{e x} \frac{\partial v_{e z}}{\partial x}+v_{e y} \frac{\partial v_{e z}}{\partial y}\right),
\end{aligned}
$$

where $\partial / \partial z=0$ is assumed because of $2 \mathrm{D}$ reconnection model.

The pressure tensor is defined as $P_{i j}=$ $m \int v_{i}^{\prime} v_{j}^{\prime} f(\vec{r}, \vec{v}, t) d \vec{v}$, where $m$ is mass, $v^{\prime}$ is random velocity, the subscript $i$ and $j$ denote the components $x$, $y$ or $z, f$ is the particle velocity distribution function, $\vec{r}$ is position, and $\vec{v}$ is velocity. In order to get the pressure tensor, number density is calculated first, from $n(\vec{r}, t)=\int f(\vec{r}, \vec{v}, t) d \vec{v}$. Mean velocity is also obtained via $\vec{V}(\vec{r}, t)=(1 / n) \int \vec{v} f(\vec{r}, \vec{v}, t) d \vec{v}$. For pressure tensor calculation, the number density, $n$ and the mean velocity, $\vec{V}(\vec{r}, t)$, is calculated by counting number of individual particles per cell and by computing the average velocity in each cell, respectively. We then estimate the random velocity, $\overrightarrow{v^{\prime}}=\vec{v}-\vec{V}$, which is used in the above definition of the pressure tensor $P_{i j}$. In PIC simulations, in practise, the summation of $m v_{i}^{\prime} v_{j}^{\prime}$ over all individual particles is used.

Figure 1 shows $y$-profiles of different terms in the generalised Ohm's law at $x=0$, for $\omega_{p e} t=170$ (timetransient reconnection peak). Here $\alpha=1.20$. Solid lines in panels (a)-(d) indicate different terms as follows: (a) $v_{e y} B_{x},(\mathrm{~b})-\left(d_{i} / n\right) \partial P_{e x z} / \partial x$, (c) $-\left(d_{i} / n\right) \partial P_{e y z} / \partial y$ and (d) $-d_{i}\left(m_{e} / m_{i}\right) v_{e y} \partial v_{e z} / \partial y$. The reconnection electric field $E_{z}(0, y)$ is shown with the dashed line in all four panels. A boxcar average scheme with a width of 7 mesh points is applied for smoothing data. The other terms in Eq. (5) are negligibly small. Fig. 11 is analogous to figure 5 from Pritchett 7]. Fig. 1(a) shows that in all regions except the magnetic null, $(0,0)$, contribution to $E_{z}(0, y)$ from the $\vec{v}_{e} \times \vec{B}$ term is significant. However, $v_{e y} B_{x}$ is zero at the X-point (the magnetic null). As seen in Fig. 1(bc), the off-diagonal components of the electron pressure tensor are major contributors to $E_{z}(0,0)$. The electron inertia term also generates the electric field away from the X-point (see Fig. 1 (d)). Thus, we conclude that the reconnection electric field is generated by the electron pressure tensor off-diagonal terms; and hence the latter are responsible for breaking the frozen-in condition. A similar conclusion was reached by Pritchett [7]. This coincidence seems somewhat unexpected, because X-point collapse considered here and onset of tearing instability considered by Pritchett [7] are physically different. Similarity of the cause of breaking of the frozen-in condition in both cases can only point to a universal nature of this mechanism. 


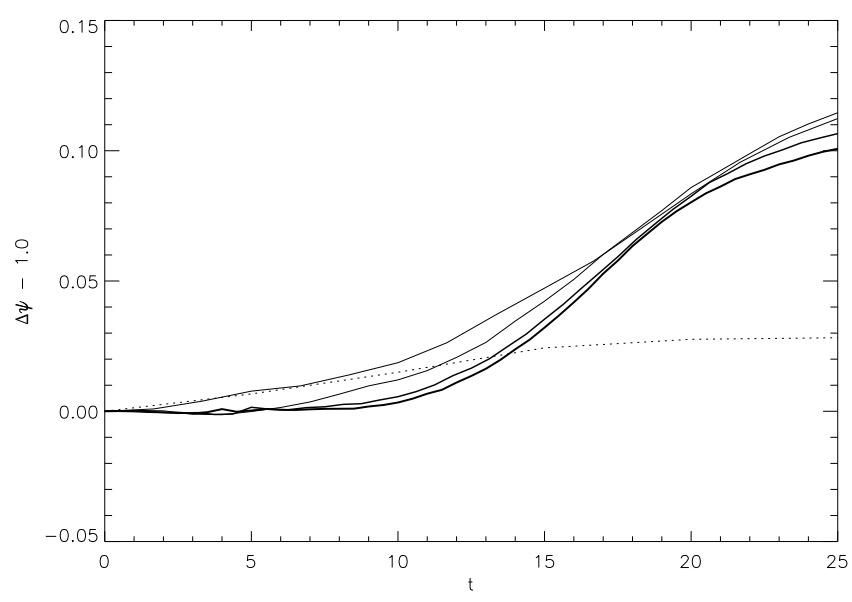

FIG. 2: Time evolution of the magnetic flux difference between the $\mathrm{O}$ and $\mathrm{X}$ points (i.e. amount of reconnected flux). The solid lines with progressively increasing thickness show cases of $m_{i} / m_{e}=9,25,64$ and 100, respectively. The dotted line shows electron-positron plasma case $\left(m_{i} / m_{e}=1\right)$. The magnetic flux difference $\Delta \psi$ is normalised by $\left(B_{0} c / \omega_{p i}\right)$ and then a unity is subtracted to start from zero. Time is normalised by the ion cyclotron frequency $\omega_{c i}=e B / m_{i}$. Here $\alpha=1.2$.

\section{B. Effect of variation of the ion-electron mass ratio and conjecture of fast reconnection}

The next question we consider is: how does the time evolution of the reconnected flux vary with the ionelectron mass ratio? Such question historically was relevant because of the inability of performing realistic ionelectron mass ratio (1836) numerical simulation, due to lack of computational resources. Although within our reach computationally, we do not show here results $m_{i} / m_{e}>100$ because the total energy conservation error (which is defined as $\left(E\left(\omega_{p e} t=250\right)-E\left(\omega_{p e} t=\right.\right.$ $0)) / E\left(\omega_{p e} t=0\right)$ and is indicative of the code accuracy) starts to deteriorate to values of circa $10 \%$ for $m_{i} / m_{e}=400$, while for $m_{i} / m_{e}=100$ it is $0.04 \%$ (both for $\alpha=1.2$ ). In order to be able to compare our results with the previous work [8], when varying $m_{i} / m_{e}$, we accordingly adjust number of spatial grid points and total time step. Such adjustments insure that spatial scale of the simulation box, is $L_{x}=L_{y}=4 c / \omega_{p i}$, and the time scale, $\omega_{c i} t=25$. Thus when setting $m_{i} / m_{e}=1,9,25,64$ and 100, Accordingly, the system size is adjusted to $40,120,200,320$ and $400 \Delta$. The global reconnection size is fixed at $L=200 \Delta$. Ion cyclotron frequency for each case is defined using by the magnetic intensity at the boundary.

In 2D the magnetic flux function can be defined as $\psi=-\int B_{x} d y=\int B_{y} d x$. In our simulation, X-point is located at the centre of system $(x, y)=(0,0)$, while $\mathrm{O}$ points are at $(x, y)=(0,-2)$ and $(0,2)$. Note that spatial coordinates here are normalised by ion skin depth, and $L_{x}=L_{y}=4 c / \omega_{p i}$. Therefore we can use the same definition of the reconnected flux as in the case tearing mode-unstable Harris current sheet [8]. Figure 2] shows time evolution of the magnetic flux difference between $\mathrm{O}$ and $\mathrm{X}$ points (reconnected flux) for different ion-toelectron mass ratios, $m_{i} / m_{e}=1,9,25,64$ and 100 . We gather from this graph that time dynamics of the reconnected flux does not depend on $m_{i} / m_{e}$ when $m_{i} / m_{e} \gg 1$ and that reconnection is fast. In fact, the time derivative of the reconnected flux is the reconnection rate. Thus, the conclusion is that the reconnection rate is independent of the mass ratio (when $m_{i} / m_{e} \gg 1$ ). As with above conclusion (in previous subsection) that the reconnection electric field is generated by the electron pressure tensor off-diagonal terms; again similarity with the tearingunstable Harris current sheet holds, i.e. Hesse et al. [8] came to the same conclusion in their case.

As a further test, we performed a numerical run with $m_{i} / m_{e}=1$ (case of electron-positron plasma). One of the main conclusions of Birn et al. [9] was that as long as Hall term is included, the reconnection is fast. i.e. when electron and ion dynamics can be distinguished. They showed that slow reconnection occurs only in the case of single fluid resistive MHD (in which there is no distinction in the electron-ion dynamics). However, in two-fluid MHD or PIC simulation it is possible to switch off the Hall term by setting $m_{i} / m_{e}=1$ as this will make electron-ion dynamics indistinguishable. The result is given by the dotted line in Fig. (2). It can be clearly seen that the amount of reconnected flux grows very slowly with time, indicating that the reconnection is slow, as expected.

We propose the following conjecture to explain why the reconnection is fast when the Hall term is included. Inclusion of the latter means that in the reconnection inflow magnetic field is frozen into electron fluid. As it was previously shown in Tsiklauri and Haruki [6] (see their Figs.(7) and (11)) speed of electrons, during the reconnection peak time, is at least 4-5 times greater than that of ions. This means that electrons can bring in / take out the magnetic field attached to them into / away from the diffusion region much faster than in the case of single fluid MHD which does not distinguish between electron-ion dynamics. In fact, in Fig.(2) the amount of reconnected flux attained by $\omega_{c i} t=25$ in the cases of $m_{i} / m_{e} \gg 1$ and $m_{i} / m_{e}=1$ has the same ratio $(0.11 / 0.03 \approx 4)$ as is the ratio of electron and ion speeds $(\approx 4-5)$.

It should be mentioned that although the importance of the Hall term for providing the fast reconnection has been firmly established, some results indicate [10, 11, 12] that the fast reconnection without it is still possible. This indicates that the issue of which physical factor(s) uniquely guarantee the fast rate is still open. 

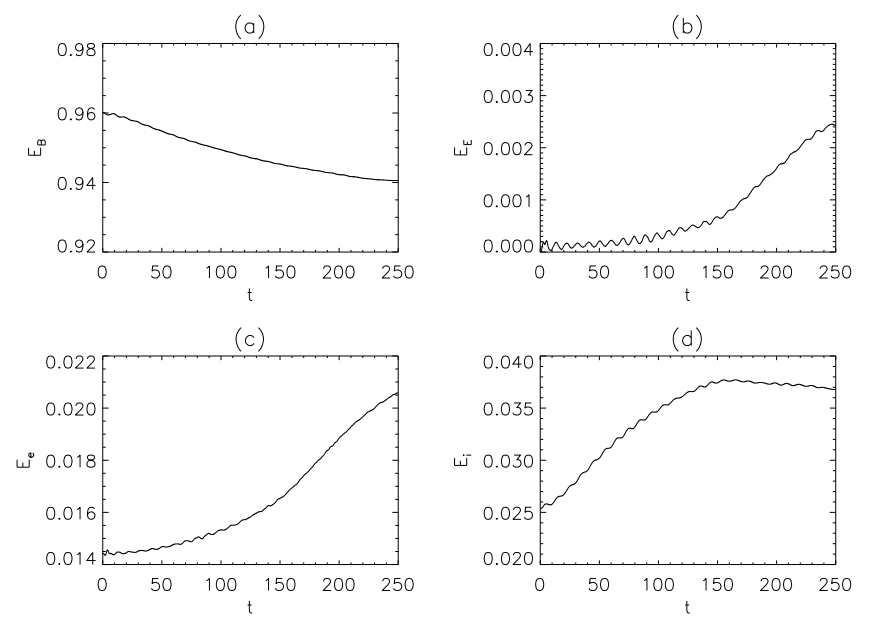

FIG. 3: Time evolution of (a) magnetic field energy, (b) electric field energy, relativistic (c) electron and (d) ion kinetic energies of the whole system for $\alpha=1.20$. These energies are normalised by the initial total energy. Time is normalised by the electron plasma frequency $\omega_{p e}$.
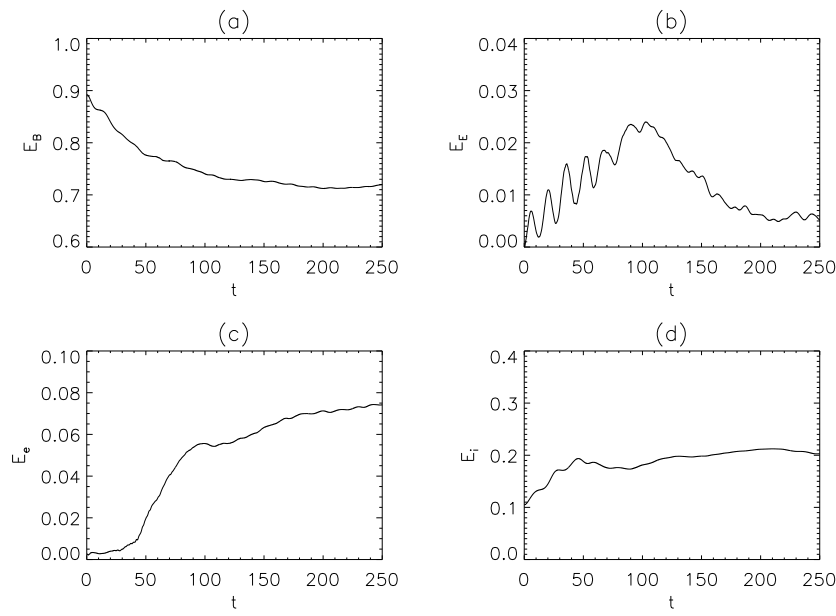

FIG. 4: The same as in Fig. 3, but for $\alpha=2.24$.

\section{Energy budget of the reconnection process}

The next question we address is: what is the exact energy budget of the reconnection process, i.e. in which proportion initial magnetic energy is converted into other forms of energy?

Figure 3 shows time evolution of (a) magnetic field energy, (b) electric field energy, relativistic (c) electron and (d) ion kinetic energies of the whole system for $\alpha=1.20$. According to the previous results [6], in this case the normalised reconnection rate peaks at $E_{z}=0.11$ at time $\omega_{p e} t=170$. Initially magnetic field energy is dominant, which constitutes $96 \%$ of the total energy of system. The rest $4 \%$ goes to the initial electron and ion kinetic energies because we impose a non-zero current $j_{z}$ at $t=0$ according to Eq.(2) (as $\alpha>1$ ). We gather from Fig. [3(a) that as the reconnection proceeds magnetic field energy is converted into other forms of energy. As it is also stated in Tsiklauri and Haruki [6] at $\omega_{p e} t=250$, which corresponds to about 1.25 Alfvén times, $(0.96-0.94) / 0.96=2$ $\%$ of the initial magnetic energy is released. Here we explore partition into which other forms this energy goes into. Panels (b)-(d) in Fig. 3 show that all other forms of energy increase as time progresses. In particular, electric field energy that starts from zero, attains value of 0.0024 , i.e. $(0-0.0024) /(0.96-0.94)=12 \%$ of the consumed magnetic energy. One can conjecture that ultimately this energy will go into particle kinetic energy (as particles would be easily accelerated by electric fields). Relativistic kinetic energy of electrons attains $(0.0205-0.0145) /(0.96-0.94)=30 \%$, while the same for ions $(0.037-0.0255) /(0.96-0.94)=58 \%$. Given that electrons have small inertia and thus are more influenced by the electric field, we conjecture that within few Alfvén times electron-ion kinetic energy partition (as the percentage of consumed magnetic energy) will be roughly $40 \%-60 \%$. It should be mentioned that, in general, it is not easy for an electric field to accelerate particles, unless it is parallel to the magnetic field or the magnetic field is small, such that the particles are non-adiabatic (loosely tied to magnetic field lines). The latter condition is more stringent for electrons. Movies of electric field in our numerical simulation show complicated, and yet coherent, oscillatory patterns. We have not performed detailed analysis of identification of nature of these waves, but based on previous experience, the case without the guide field considered here, would support excitation of whistler waves as the X-point collapses. In turn, we conjecture that the whistler waves are ultimately responsible for the particle acceleration. More detailed analysis of this is needed in the future. Returning to the issue of the established 40\% - 60\% energy partition, Emslie et al. [13] showed that the energy of accelerated electrons is comparable to that of accelerated ions. However, they admit to large uncertainties in the ion energy spectrum. Despite of this, our simulation results broadly agree with the solar flare observations [13].

Previously we also considered strongly stressed X-point $(\alpha=2.24)$ [6]. In this case in 1.25 Alfvén times, $(0.9-0.72) / 0.9=20 \%$ of the initial magnetic energy is converted into other forms of energy (this is equivalent of $(0.9-0.72)=18 \%$ of the initial total energy; and as we saw in the weakly stressed case, the difference between the two is negligible. It is only with the increase of $\alpha$ the difference between initial magnetic energy and initial total energy becomes noticeable, because stronger initial currents (i.e. initial kinetic energy of particles) need to be imposed according to Eq.(21)). This 18\% decrease in the magnetic energy is also corroborated in panel (a) in Fig. 4. Exact break down (partition) of the latter is as follows (based on panels (b)-(d)): electric field energy that starts from zero, peaks and then settles at 0.006 , i.e. $(0-0.006) /(0.9-0.72)=3 \%$ of the consumed magnetic energy. Relativistic kinetic energy of electrons attains 

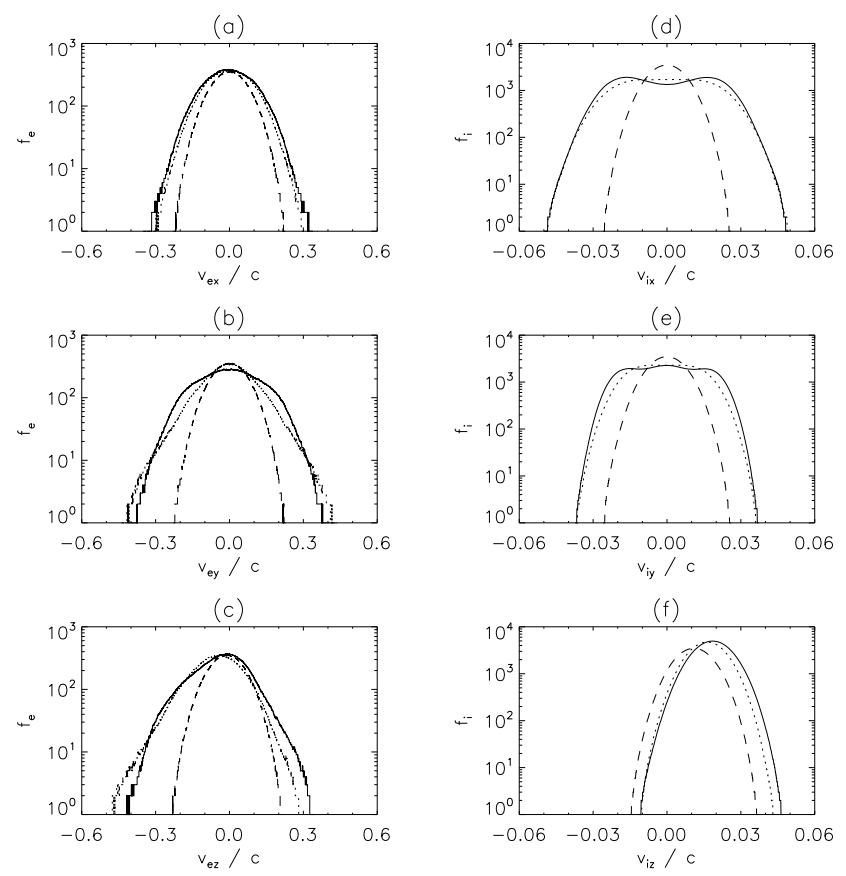

FIG. 5: (a-c) Electron and (d-f) ion velocity distribution functions in $x, y$ and $z$ directions near the current sheet at the initial stage $t=0$ (dashed line), the peak reconnection stage $t=170$ (dotted line) and the final simulation time $t=250$ (solid line) for $\alpha=1.20$. As in Ref.[6], here data are produced using the region of the current sheet $\left(-2\left(c / \omega_{p e}\right) \leq x \leq 2\left(c / \omega_{p e}\right),-8\left(c / \omega_{p e}\right) \leq y \leq 8\left(c / \omega_{p e}\right)\right) . f_{e}$ and $f_{i}$ are the number of electrons and ions, respectively. Velocity and time are normalised by light speed $c$ and $\omega_{p e}$, respectively.

$(0.075-0.002) /(0.9-0.72)=41 \%$, while the same for ions $(0.2-0.1) /(0.9-0.72)=56 \%$. As in the weakly stressed case, within 1.25 Alfvén times, electron-ion kinetic energy partition (as the percentage of total energy, which for a solar flare would be the total energy released by flare) is roughly $40 \%-60 \%$. This again is in accord to solar flare observations Emslie et al. [13].

\section{Properties of velocity distribution of the accelerated particles}

The final question we address is: are there any anisotropies in the velocity distribution of the accelerated particles? This question naturally comes to one's mind due to a recent study of Kontar and Brown [14], who surprisingly found near-isotropic electron distributions in solar flares, which contrast strongly with the expectations from the standard model that invokes strong downward beaming, including the collisional thick-target model.

As in Tsiklauri and Haruki [6], here we consider two cases of the weakly and strongly stressed X-point. The results are shown in Figures [5] and 6] The following ob-
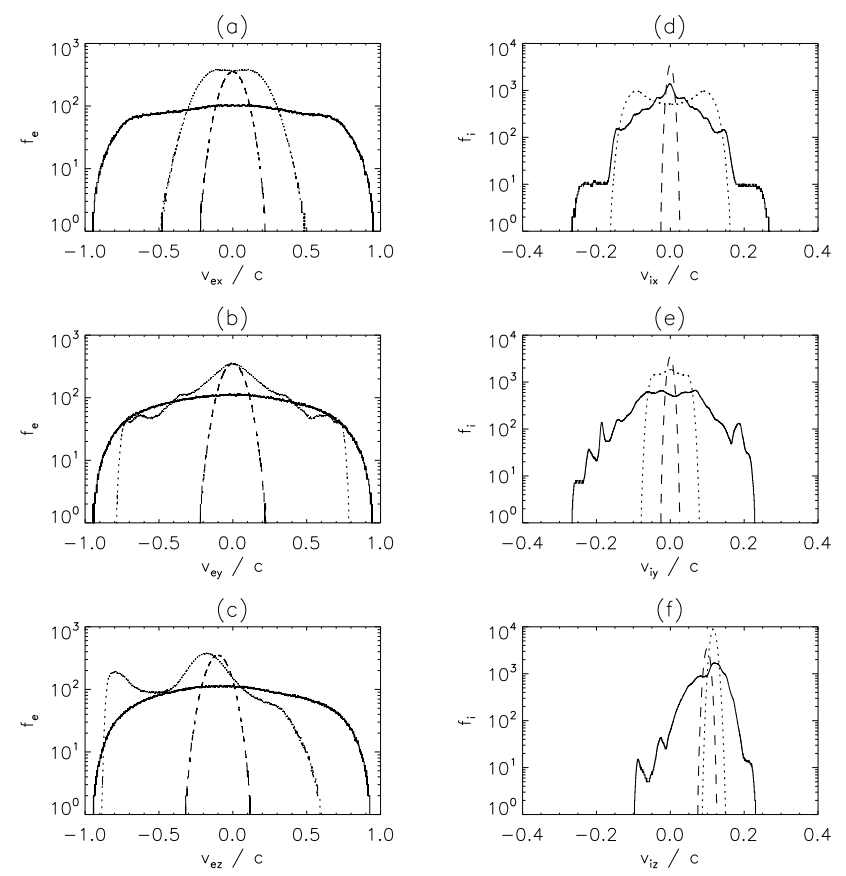

FIG. 6: (a-c) Electron and (d-f) ion velocity distribution functions in $x, y$ and $z$ directions near the current sheet at the initial stage $t=0$ (dashed line), the peak reconnection time $t=45$ (dotted line) and the final simulation time $t=250$ (solid line) for $\alpha=2.24$. Data are produced using the region of the current sheet $\left(-1\left(c / \omega_{p e}\right) \leq x \leq\right.$ $\left.1\left(c / \omega_{p e}\right),-16\left(c / \omega_{p e}\right) \leq y \leq 16\left(c / \omega_{p e}\right)\right)$. The normalisation is same as in Fig [5.

servations can be made:

In the weakly stressed case, for electrons we see appearance of super-thermal electrons towards the end of simulation time (shortly after the peak of time-transient reconnection) mostly in $y$ and $z$ velocity distribution function components. Dynamics of the flows and currents is presented in detail in Ref.[6]. Here we only mention that the reconnection inflow is in $x$ direction, while the outflow is in $y$ direction. Thus based on panels (a)-(c) in Fig. (5) we gather that accelerated electrons (focus on solid and dotted curves) are due to reconnection outflow (in reconnection plane) as well as out-of-plane flow (which is triggered by the out-of-plane electric field generated at the magnetic null). For ions, at later stages of the reconnection, in panels (d)-(f) in Fig.(5) we see (focus on solid and dotted curves) a superposition of two Maxwellian distributions in both reconnection inflow (along $x$ ) and outflow (along $y$ ). These seem to be created by reconnection flow dynamics. In $z$-direction we see a shifted (also somewhat broadened by the heating) Maxwellian, which is due to out-of-plane ion beam (localized current).

We gather from panels (a)-(b) in Fig.(6) (focus now only on solid curves) that in the strongly stressed X-point case, in about one Alfvén time, super-thermal electrons show a full isotropy in all three spatial directions of the velocity distribution. In solar flare observations Kon- 
tar and Brown [14] report that electron distributions are also nearly isotropic, which seems to contradict to what is expected from the standard model flare models that invoke strong downward beaming of electrons. In this respect, the match of our simulation results with the flare observations seem encouraging, in that stressed X-point collapse seems to be a viable mechanism acting during solar flares. For ions (panels (d)-(f) in Fig.(6) ) behaviour is not so much different from the weakly stressed case (panels (d)-(f) in Fig.(5)), except for much higher velocities attained and distribution functions modified by kinetic, wave-particle interaction instabilities. The latter can be judged by sign changes in the slope of the distribution function, which can only occur when waves and particles exchange energy and momentum.

\section{CONCLUSIONS}

By and large, the present work closes our initial study of stressed X-point collapse in the collisionless regime started in Ref. [6], by bridging gaps in the understanding of key physical aspects. The main findings can be listed as following:

(i) despite significant differences of the initial setup between tearing unstable Harris current sheet [7] and stressed X-point considered here, in both cases source of the reconnection out-of-plane electric field at the magnetic null is provided by off-diagonal terms of the electron pressure tensor.

(ii) we find that when $m_{i} / m_{e} \gg 1$ reconnection rate is independent of the ion-electron mass ratio and it is fast, which is also witnessed by Hesse et al. [8]. However, when electron-ion mass ratio is unity, i.e. the Hall term is switched off, we show that reconnection rate is indeed slow. This broadly agrees with the results of Birn et al. [9] (However see also Refs. 10, 11, 12] for alternative view). When the Hall physics is included, we also conjecture that the reconnection is fast because the magntic field (being frozen into electron fluid, which moves significantly faster than ion fluid, as shown in Ref.[6]) is transported in and out of the diffusion region much faster than in the case of single fluid resistive MHD. We show that the amount of reconnected flux attained by $\omega_{c i} t=25$ in the cases of $m_{i} / m_{e} \gg 1$ and $m_{i} / m_{e}=1$ has the same ratio $(\approx 4)$ as is the ratio of electron and ion speeds $(\approx 4-5)$.

(iii) we find that within one Alfvén time, roughly $40 \%$ of the initial total energy (which is mostly stored in the magnetic field) is converted into the kinetic energy electrons, and somewhat more than half $(\sim 60 \%)$ into kinetic energy of ions. In solar flare observations a similar behaviour is seen [13].

(iv) When X-point is stressed strongly, in about one Alfvén time, a full isotropy in all three spatial directions of the velocity distribution is seen for super-thermal electrons. Again similar behaviour is reported in solar flare observations 14.

Resuming aforesaid, it seems that collisionless, stressed $\mathrm{X}$-point collapse is a viable mechanism for solar flares. Also, its behaviour is remarkably similar to tearing unstable Harris current sheet which is thought to be more relevant for the Earth geomagnetic tail and generally to magnetospheric applications.

We close this paper with some words of caution:

(i) realising that size of the numerically simulated area $40 \times 40$ electron skin depths $\left(c / \omega_{p e}=0.16804 \mathrm{~m}\right.$ in solar corona), i.e. $6.7 \times 6.7 \mathrm{~m}$ is a tiny proportion of the actual solar flare site which can be tens of Mm. Thus, $a$ priori it is not clear whether this mechanism is suitable to accelerate enough electrons in a much larger volume.

(ii) whether e.g. the obtained electron-ion kinetic energy partition (as the percentage of total released energy) being roughly $40 \%-60 \%$, would hold for a different mass ratio? We remind the reader that except in subsection III.B the ion-electron mass ratio was kept constant at $m_{i} / m_{e}=100$. We can only conjecture that this $40 \%$ - $60 \%$ partition holds for a different mass ratio, because we also found that the amount of reconnected flux versus time graph (Fig. 2) showed no mass ratio dependence.

\section{Acknowledgments}

This research was supported by the United Kingdom's Science and Technology Facilities Council (STFC).
[1] D. Biskamp, Magnetic reconnection in Plasmas (Cambridge University Press, 2005).

[2] J. D. Huba, NRL plasma formulary (Naval Research Laboratory, Washington DC 20375, 2004).

[3] J. Birn and E. R. Priest, Reconnection of magnetic fields: magnetohydrodynamics and collisionless theory and observations (Cambridge University Press, 2007).

[4] J. W. Dungey, Phil. Mag. 44, 725 (1953).

[5] E. Priest and T. Forbes, Magnetic reconnection: $M H D$ theory and applications (Cambridge University Press, 2000).

[6] D. Tsiklauri and T. Haruki, Physics of Plasmas 14,
112905 (2007).

[7] P. L. Pritchett, J. Geophys. Res. 106, 3783 (2001).

[8] M. Hesse, K. Schindler, J. Birn, and M. Kuznetsova, Physics of Plasmas 6, 1781 (1999).

[9] J. Birn, J. F. Drake, M. A. Shay, B. N. Rogers, R. E. Denton, M. Hesse, M. Kuznetsova, Z. W. Ma, A. Bhattacharjee, A. Otto, J. Geophys. Res. 106, 3715 (2001).

[10] N. Bessho and A. Bhattacharjee, Physics of Plasmas 14, 056503 (2007).

[11] W. Daughton and H. Karimabadi, Physics of Plasmas 14, 072303 (2007).

[12] M. Hesse and S. Zenitani, Physics of Plasmas 14, 112102 
(2007)

[13] A. G. Emslie, H. Kucharek, B. R. Dennis, N. Gopalswamy, G. D. Holman, G. H. Share, A. Vourlidas, T. G. Forbes, P. T. Gallagher, G. M. Mason, Journal of Geo- physical Research (Space Physics) 109, 10104 (2004).

[14] E. P. Kontar and J. C. Brown, Astrophys. J. Lett. 653, L149 (2006). 